\title{
GENERACIÓN DE ACTITUDES FAVORABLES \\ HACIA LAS CIENCIAS \\ DESDE UNA PERSPECTIVA DE \\ ENSEÑANZA-APRENDIZAJE POR INVESTIGACIÓN
}

\author{
Carmen Cecilia Cortés Rubiano dqu920- ccortes@pedagogica.edu.co \\ Martha Patricia Rodríguez Chagualá dqu956 mrodriguez@pedagogica.edu.co
}

\section{RESUMEN}

El presente trabajo busca mostrar la importancia de los proyectos en el aula y la relación de la química con la vida cotidiana, buscando así, promover la investigación en la educación básica, de tal modo que se utiliza el modelo enseñanza-aprendizaje por investigación como herramienta metodológica, el cual permitió a los estudiantes adquirir habilidades de interpretación, argumentación y sobre todo, de relación y participación. Lo anterior posibilita al estudiantado proponer fenómenos cotidianos y entender su relación con la química gracias a los proyectos desarrollados.

\section{PALABRAS CLAVE}

Enseñanza-aprendizaje, aprendizaje por investigación, proyectos, PGA, tratamiento de agua potable

\begin{abstract}
This paper aims to show the importance of classroom projects and the relationship of chemistry to everyday life, looking well, promote research in basic education, so that using the teaching-learning model as a tool for research methodology, which allowed students to acquire skills of interpretation, argument and above all, respect and participation. This enables the students to propose everyday phenomena and understand their relationship with chemistry thanks to the projects developed.
\end{abstract}

Finally determined as these activities generate favorable or unfavorable attitudes of students towards science and in particular to the chemical.

\section{Keywords}

Teaching-learning, learning research, projects, PGA, drinking water treatment 


\section{INTRODUCCIÓN.}

A lo largo del tiempo se ha identificado, que la enseñanza de las ciencias ha optado por un modelo tradicional, lo que conlleva a que los estudiantes no posean motivación alguna por dicha disciplina, así mismo, se observa las fallas que los estudiantes tienen al momento de interpretar, argumentar y proponer una problemática del diario vivir, dando como resultado la incapacidad de responder a los retos de la vida diaria.

Por esto, se hace necesario incentivar al estudiantado a relacionarse con los fenómenos, de tal manera que adquieran un interés propio hacia la química. De modo que La educación en ciencias no solo debe promover el aprendizaje de conceptos científicos, sino también, debe abarcar la formación de actitudes e intereses favorables hacia la ciencia y en particular hacia la química, para que en el individuo adquiera habilidades participativas, argumentativas propositivas e interpretativas y a su vez desarrolle habilidades y capacidades para comprender situaciones de su cotidianidad.

En este orden de ideas se inició con una serie actividades donde se buscó que los estudiantes se generaran preguntas del ¿por qué de los hechos? A partir de estas situaciones iníciales, el trabajo práctico pedagógico se basa en el modelo didáctico para la enseñanza-aprendizaje de la química por investigación.

El uso de experiencias, casos y demás relacionados con la vida cotidianidad, es un vía pertinente para abordarse las temática de la química y realizar actividades donde el estudiantado proponga posibles alternativas de solución para una situación problema como lo es: El tratamiento de agua potable, ya que es un recurso que está al alcance de todos los seres humanos y a su vez es indispensable para el diario vivir, pero poco se conoce de su tratamiento para el consumo humano, por ello se selecciona esta temática para introducirla en el curso de química de grado décimo.

\section{DESCRIPCIÓN DEL PROBLEMA}

En el período de observación e identificación del problema se determinó que los estudiantes de grado décimo, de colegio liceo nacional Antonia santos, presentan gran dificultad para interpretar y dar solución a problemas cotidianos de la vida diaria desde la química, dando como resultado el desagrado por la disciplina al no poder establecer la relación de lo aprendido con lo vivido. En este sentido se formula la siguiente pregunta problema:

¿Es posible que un modelo de enseñanzaaprendizaje, centrado en investigación, permita que los estudiantes relacionen fenómenos de la vida cotidiana desde la química y favorezca sus actitudes hacia esta?

\section{MARCO TEÓRICO}

Para el desarrollo de esta propuesta se hace necesario definir algunos términos relevantes relacionados con el modelo enseñanza aprendizaje por investigación y algunos antecedentes que permiten tener una visión más clara de lo que se llevo a cabo.

\section{ENSEÑANZA-APRENDIZAJE POR INVESTIGACIÓN.}

La idea central del modelo de aprendizaje de las ciencias como investigación que se propuso consiste, como acabamos de ver, en el tratamiento de situaciones problemáticas abiertas de interés, a través de las cuales lo alumnos puedan participar en la construcción de los conocimientos. Una primera cuestión que ello plantea, recordemos, es la de hasta qué punto los alumnos pueden (re) construir unos conocimientos que tanto tiempo y esfuerzos han exigido a los científicos más notables. (Gil. Pérez, D. 1992) 
Este modelo didáctico de carácter alternativo se propone como finalidad educativa el "enriquecimiento del conocimiento de los alumnos" en una dirección que conduzca hacia una visión más compleja y crítica de la realidad, que sirva de fundamento para una participación responsable en la misma. Se adopta en él una visión relativa, evolutiva e integradora del conocimiento, de forma que en la determinación del conocimiento escolar constituye un referente importante el conocimiento disciplinar. (Francisco F. García Pérez 2000)

\section{PROGRAMA GUÍA DE ACTIVIDA- DES}

"Los Programas Guía son propuestas de desarrollo de unidades didácticas, se conciben como un conjunto de actividades con una secuencia lógica y en orden creciente de dificultad que se van intercalando con información que el estudiante va consultando. Para trabajar con los Programas Guías de Actividades
(PGA), los estudiantes deben realizar una tarea ordenada para abordar el trabajo propuesto. El maestro juega el papel de guía del aprendizaje teniendo especial cuidado que las actividades no supongan pequeños trabajos aislados e inconexos. (Rodríguez ,J. Hernández, D . Castrillón, W. 2009)

\section{INVESTIGACIÓN DIRIGIDA}

La investigación dirigida es una actividad experimental que requiere la participación activa del estudiante y que orienta la búsqueda de una evidencia que permita resolver un problema práctico o contestar un cuestionamiento teórico. Este es un proceso de indagación que usualmente lo realiza el estudiante en forma individual o grupal, luego que el maestro asigna un trabajo, y para lograr el cumplimiento de los objetivos, proporciona a los estudiantes una guía, para que ellos la manejen y puedan realizar su proyecto. (Mora. A 2005) 
Teniendo en cuenta estos conceptos claves, se da paso al referente teórico más importante de este trabajo, se le da la connotación importante ya que es el antecedente encontrado acerca de la propuesta realizada. A continuación se presenta un esquema del artículo:

\section{"MODELO DIDACTICO PARA EL APRENDIZAJE DE LA QUIMICA BASICA CON ALUMNOS DE BAJO DESEMPENO" \\ Chamizo J.A., Sosa P., Sosa}

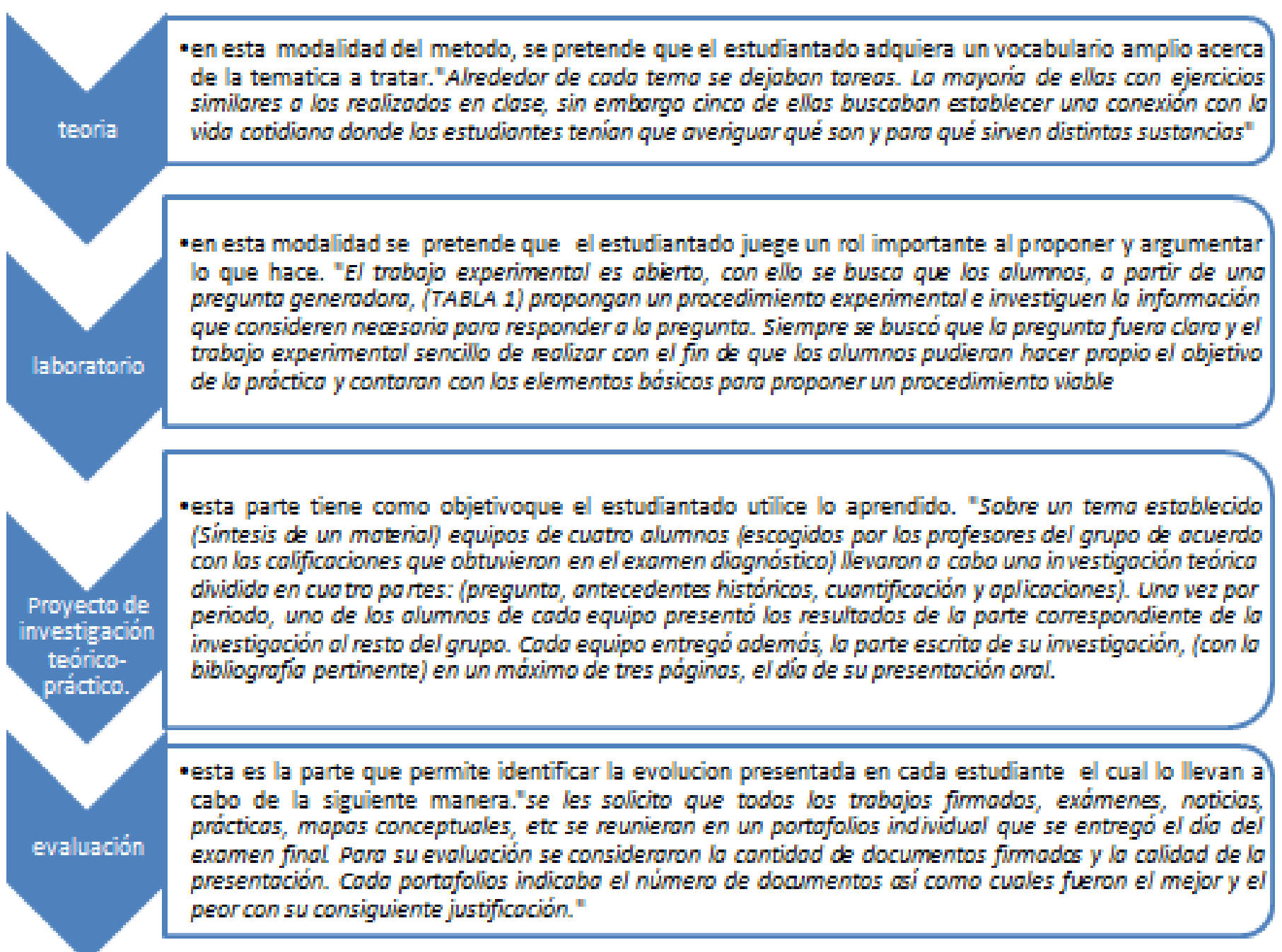


- Comprobar si el modelo pedagógico y didáctico de enseñanza-aprendizaje por investigación resulta ser apropiado para incentivar, motivar al estudiantado y así mismo identificar como el modelo afecta favorablemente o desfavorablemente las actitudes de los estudiantes hacia las ciencias, y en particular de la química.
-Identificar las ventajas y desventajas del modelo para favorecer las actitudes de los estudiantes hacia la química.

-Indagar si los estudiantes adquieren mayor habilidad para explicar los fenómenos de la vida cotidiana desde la química.

- Elaborar e implementar instrumentos que permitan identificar el desarrollo intelectual $y$ actitudinal que tienen los estudiantes antes y después del la incorporación del modelo

\section{METODOLOGÍA}

\begin{tabular}{|c|c|}
\hline Datos de identificación: & $\begin{array}{l}\text { Colegio: liceo nacional Antonia santos J.M. } \\
\text { Grado: decimo ( } 10.01 \text { y } 10.03 \text { ) } \\
\text { Número de estudiantes: } \\
\text { Control: } 31 \\
\text { Piloto: } 12 \\
\text { Total: } 43 \\
\end{array}$ \\
\hline $\begin{array}{l}\text { Técnicas e instrumentos } \\
\text { de recolección de infor- } \\
\text { mación : }\end{array}$ & $\begin{array}{l}\text { Guías de laboratorio. } \\
\text { Prácticas de laboratorio. } \\
\text { Avances de los proyectos. } \\
\text { Test de actitudes hacia la ciencia. }\end{array}$ \\
\hline
\end{tabular}

\section{ESTRATEGIA METODOLÓGICA}

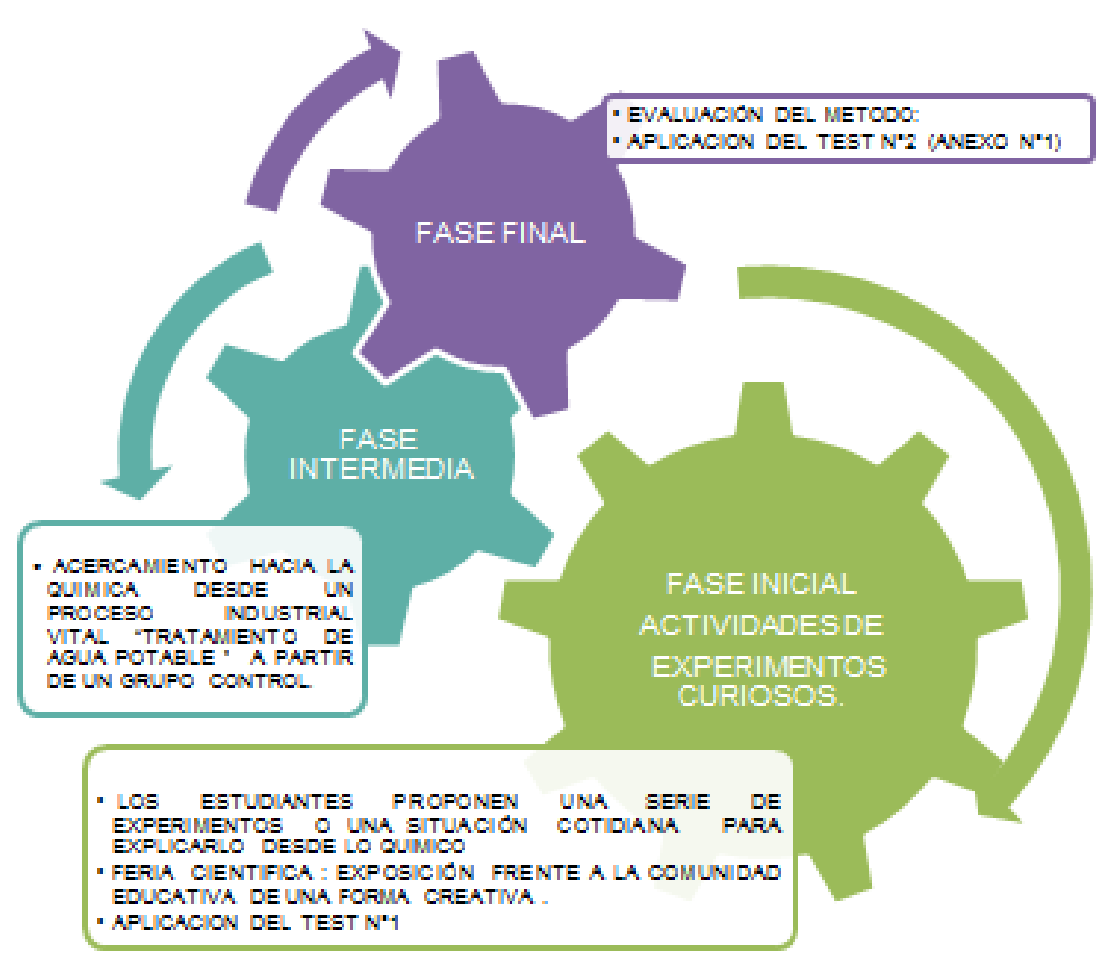


Se hace necesario destacar, que en la fase intermedia, al grupo piloto de estudiantes se le dio el nombre de "grupo de investigación ", al cual se le aplica la estrategia metodológica, la que se describe a continuación:

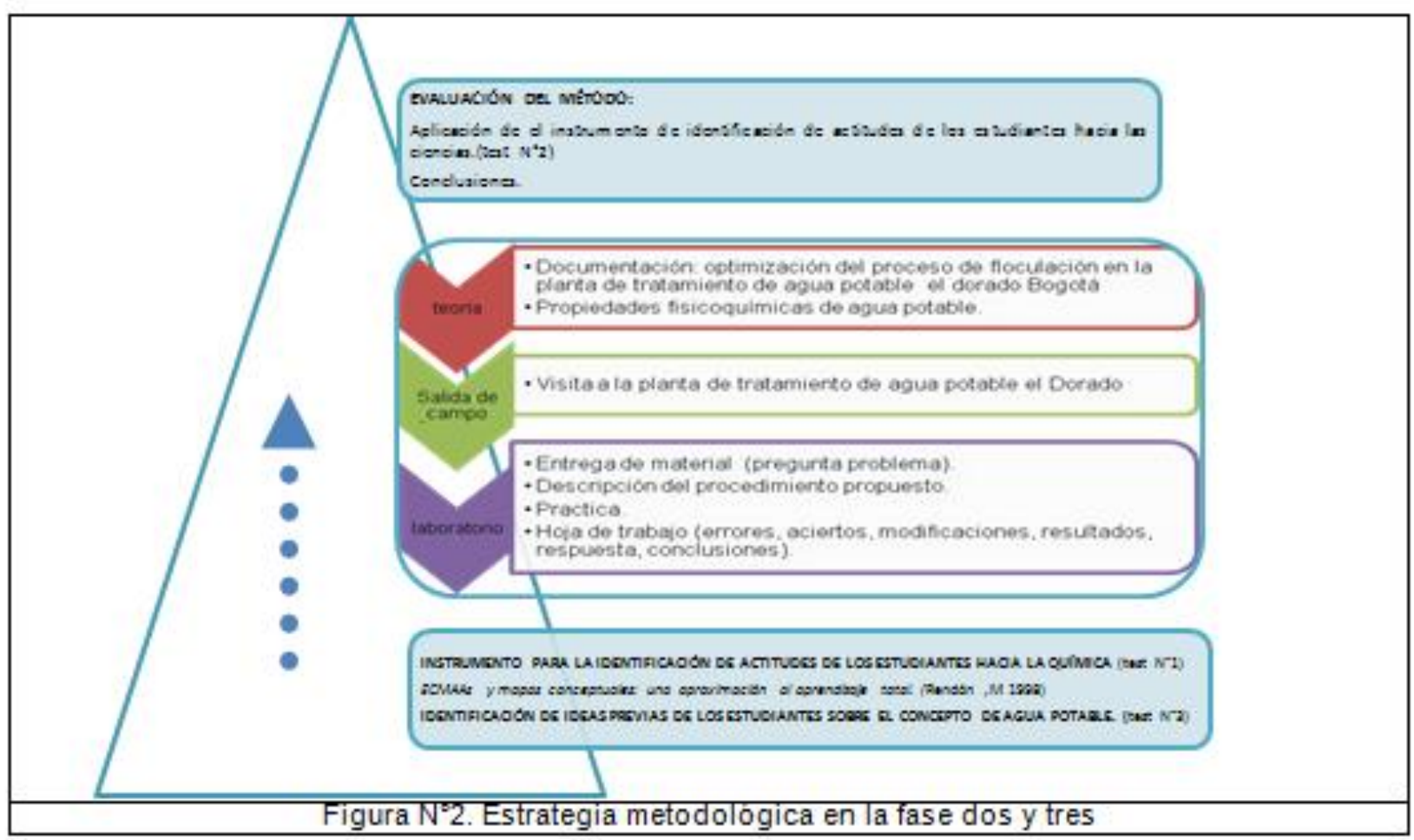

RESULTADOS

La puntuación de la escala de valores implementada, consiste en sumar algebraicamente los valores asignados por los estudiantes para los ítems $(5,8,10,12,17,18,20)$ donde la puntuación 28 es la más favorable hacia la química.

Para la cuantificación de los resultados obtenidos en este test se hizo necesario realizar un paralelo con las preposiciones no favorables hacia la química como son los ítems $(2,4,9,11,15,16,19)$, de tal modo que la suma algebraica para estos ítems es 119 lo cual indica una actitud muy desfavorable hacia la química. Por lo tanto se realizo un intervalo en el cual se clasificaron los puntajes obtenidos de los estudiantes, de este modo el intervalo para actitudes favorables es 28 hasta 59 , para actitudes neutras el intervalo oscila 60 hasta 87 , y para actitudes desfavorables está entre 88 hasta 119. A continuación se reportan los resultados obtenidos 


\begin{tabular}{|c|c|c|c|}
\hline \multicolumn{4}{|c|}{ GRUPO DE INVESTIGACION “PILOTO” } \\
\hline $\begin{array}{c}N^{\circ} \text { ESTU- } \\
\text { DIANTES }\end{array}$ & $\begin{array}{c}\text { PUNTAJE } \\
\text { DESFAVO- } \\
\text { RABLE }\end{array}$ & $\begin{array}{c}\text { PUNTAJE } \\
\text { NEUTRO }\end{array}$ & $\begin{array}{c}\text { PUNTAJE MUY } \\
\text { FAVORABLE }\end{array}$ \\
\hline 1 & & 72 & 49 \\
\hline 2 & & 79 & \\
\hline 3 & & & 36 \\
\hline 4 & & & 58 \\
\hline 5 & & & 50 \\
\hline 6 & & & 46 \\
\hline 7 & & & 58 \\
\hline 8 & & 76 & \\
\hline 9 & & & \\
\hline 10 & 83 & & 7 \\
\hline 11 & & 65 & \\
\hline 12 & & & \\
\hline TOTAL & 1 & 4 & \\
\hline
\end{tabular}

Tabla 1. Actitudes de los estudiantes, del grupo piloto, hacia la química

\begin{tabular}{|c|c|c|c|}
\hline \multicolumn{4}{|c|}{ GRUPO CONTROL } \\
\hline $\begin{array}{l}N^{\circ} \text { ESTU- } \\
\text { DIANTES }\end{array}$ & $\begin{array}{l}\text { PUNTAJE DES- } \\
\text { FAVORABLE }\end{array}$ & $\begin{array}{l}\text { PUNTA- } \\
\text { JE NEU- } \\
\text { TRO }\end{array}$ & $\begin{array}{l}\text { PUNTAJE } \\
\text { MUY FAVO- } \\
\text { RABLE }\end{array}$ \\
\hline 13 & 104 & & \\
\hline 14 & & 67 & \\
\hline 15 & 101 & & \\
\hline 16 & 114 & & \\
\hline 17 & & 79 & \\
\hline 18 & & 86 & \\
\hline 19 & & 61 & \\
\hline 20 & & & 57 \\
\hline 21 & 99 & & \\
\hline 22 & 101 & & \\
\hline 23 & & 75 & \\
\hline 24 & 96 & & \\
\hline 25 & & 63 & \\
\hline 26 & 112 & & \\
\hline 27 & 109 & & \\
\hline 28 & & 61 & \\
\hline 29 & & 75 & \\
\hline 30 & & 75 & \\
\hline 31 & & 64 & \\
\hline 32 & & 82 & \\
\hline 33 & & 78 & \\
\hline 34 & & 81 & \\
\hline 35 & & 68 & \\
\hline 36 & 109 & & \\
\hline 37 & 111 & & \\
\hline 38 & 119 & & \\
\hline 39 & 116 & & \\
\hline 40 & & 65 & \\
\hline 41 & & 78 & \\
\hline 42 & & & 55 \\
\hline 43 & 106 & & \\
\hline TOTAL & 13 & 16 & 2 \\
\hline
\end{tabular}

Tabla 2. Actitudes de los estudiantes, del grupo control, hacia la química

Gráfica 1. Actitudes, hacia la química de los estudiantes, del grupo de investigación. 


\section{ANÁLISIS DE RESULTADOS}

Resulta necesario analizar y contrastar los resultados obtenidos del grupo control y del grupo piloto, por consiguiente se reportan las gráficas correspondientes con su respectivo análisis para cada grupo.

Como se puede observar en la Gráfica 1, se evidencia que del total de los estudiantes del grupo de investigación, el $59 \%$ presenta una actitud favorable hacia la química, siendo este un porcentaje favorable en comparación a lo obtenido en el test $\mathrm{N}^{\circ} 1$, sin embargo, se evidencia un porcentaje alto para actitudes neutras, dando así prioridades a otras aéreas de conocimiento, marcando una gran afinidad por la parte de artes, sin ser indiferentes con los saberes científicos y en particular químicos y finalmente, se evidencia un porcentaje considerablemente pequeño que posee actitudes desfavorables hacia la química.

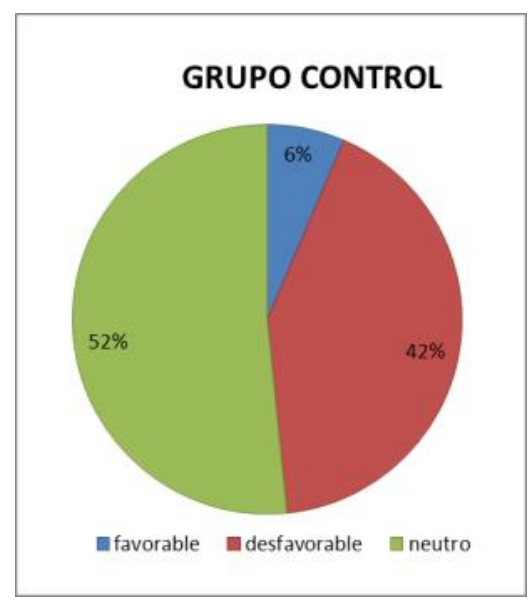

Gráfica 2. Actitudes, hacia la química, de los estudiantes del grupo control

Observando los resultados obtenidos se hace evidente que los estudiantes presenta mayor afinidad por otros campos del saber dando como resultado una alta indiferencia al saber químico ya que se obtiene el cuarenta y dos por ciento de estudiantes con actitudes desfavorables hacia la química .sin embargo se reporta que el 52 por ciento de los estudiantes poseen una actitud neutra, lo que indica que la química no es les el del todo indiferente. Finalmente se eviden- cia que seis por ciento tienen actitudes favorables hacia la química, siendo este un resultado muy desalentador para la ciencia en general.

Se realizó el paralelo entre los resultados obtenidos para cada uno de los grupos teniendo en cuenta los porcentajes favorables y desfavorables hacia la química en donde se reporta el resultado final de la propuesta pedagógica y didáctica planteada para favorecer las actitudes de los estudiantes hacia la ciencia.

En la gráfica 3 , se observa la diferencia marcada entre los grupos de estudio frente a las actitudes hacia la ciencia.

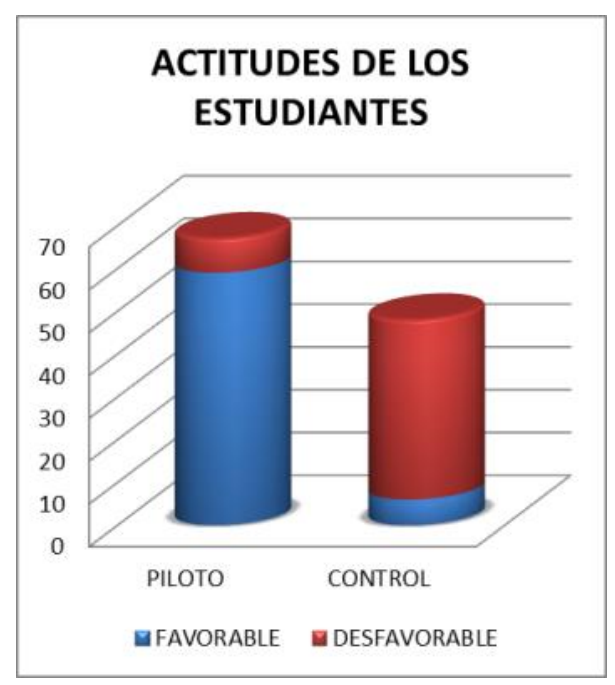

Gráfica 3. Comparación de las actitudes, hacia la química, de los grupos en estudio

El modelo implementado permite adquirir un actitud positiva hacia la ciencia y particular hacia la química. Obteniéndose una diferencia alta en cuanto a los porcentajes favorables y desfavorables hacia la ciencia entre los dos grupos.

Finalmente se puede deducir que la propuesta pedagógica y didáctica planteada, desde el modelo enseñanza aprendizaje por investigación, permite generar actitudes favorables hacia la química. 


\section{CONCLUSIONES}

-El objetivo general se cumple porque se evidenció que la enseñanza -aprendizaje de la química al relacionarlo con la vida cotidiana despertó el interés hacia la química favoreciendo las actitudes del los estudiantes en ambos grupos como se observa en la gráfica $\mathrm{N}^{0} 1$ y gráfica $\mathrm{N}^{0} 2$ donde se obtiene el $59 \%$ de actitud favorable con el grupo piloto y el $6 \%$ con el grupo control.

- Este proyecto, lo mismo que el trabajo realizado por-Chamizo J.A., Sosa P., Sosa A. "Modelo didáctico para el aprendizaje de la química básica con alumnos de bajo desempeño facultad de química, universidad nacional autónoma de México" permitió que el estudiante desarrolle competencias de interpretación, argumentación y proposición dando como resultado actitudes favorables hacia la química.
- La implementación de salidas pedagógicas y didácticas en el área de química relacionada con procesos industriales resultó motivante para los estudiantes promoviendo así su interés por el saber químico y su actitud como se muestra en la gráfica 3 al realizar el paralelo entre el grupo piloto y control. A partir de relación teórica - práctica en la enseñanza aprendizaje de la química que se realizó permitió g generar habilidades y actitudes favorables hacia la química obteniéndose los resultados del test 3 el valor menor para los ítems. $(5,8,10,12$, $17,18,20)$ que se relacionan directamente con la química. Como se reporta en las tabla 1 y 2.

\section{BIBLIOGRAFÍA}

* Chamizo J.A., Sosa P., Sosa A. Modelo didáctico para el aprendizaje de la química básica con alumnos de bajo desempeño facultad de química, universidad nacional autónoma de México. 04510 México D.F., México de, http://ww.joseantoniochamizo.com/ pdf/0201_46.pdf. Recuperado el 15 de julio de 2012 .

* Francisco F. García Pérez (2000, Febrero 18). Los modelos didácticos como instrumento de análisis y de intervención en la realidad educativa .Biblio $3 W$. Revista Bibliográfica de Geografía y Ciencias Sociales .Universidad de Barcelona 207 pp.6-7

* Gil, Pérez D. (1992). Contribución de la historia y la filosofía de las ciencias al desarrollo de un modelo de ense- ñanza-aprendizaje como investigación. Enseñanza de las Ciencias, 11(2), pp. 197-212.

* Mora, A, Investigación dirigida. VII congreso nacional de ciencias Exploraciones fuera $y$ dentro del aula. 2005,Agosto 26-27 Costa rica.pp.1-2

*Rodríguez,J.Hernández,D.CastrillónW .(2009) Diseño de un programa guía de actividades para la enseñanza de la química en educación media basado en el modelo didáctico por investigación dirigida. I congreso Nacional de investigación en educación en ciencias y tecnología Asociación Colombiana para la investigación en Ciencias $Y$ Tecnología EDUCyT, Memorias 2009, Junio 22 a 26.pp 2 